DOI: 10.20472/IAC.2018.042.010

\author{
ADAM FEDYNIUK \\ Nicolaus Copernicus University, Poland
}

\title{
TECHNOLOGICAL SINGULARITY: A CONNECTOMICS PERSPECTIVE
}

\begin{abstract}
:
There are many definitions, approaches and models of technological singularity. In most cases it can be summarized as "changes in the mode of human life, which gives appearance of approaching some essential singularity in the history of human race, beyond which human affairs, as we know them, could not continue" 1 . When considering the possibility of technological singularity in the form of emergence of superintelligence, we are given also some variety in its facets like, self-improving technology, accelerating change or simply put, intelligence explosion2. These forms vary in the antecedents that define the initial state of affairs that would become the foundation of the arrival of singularity. The debate concerning this hypothetical phenomenon can be polarizing, with no consensus on the horizon. Even when we take into account cognitive science, as the basis for formulation of possible paths technological progress can take, and result in a singularity, there can be a stern critique3. Still, with a balance between enthusiasm and critique, being rooted in constructive approach to this idea, we can make viable attempt at better understanding and predicting what future may hold for the human race4. Comparably, with the advent of connectomics and the advancement of studies on large-scale networks we can make even more detailed attempt at explaining and modelling possible emergence of artificial general intelligence. Especially, due to how we can of define and model emergent properties that such biological organisations possess 5 . The methods, with which we can view, analyze and discover properties of hierarchical structures can lead the way into a more detailed view of cognition. There is also the possibility of emulating it on a more robust platform (self-improving array of integrated circuits, adiabatic processors and similar). we have the possibility to design Al that will spark a paradigm-shift for the future research and our understanding of mind.
\end{abstract}

\section{Keywords:}

technological singularity, connectomics, network theory, complexity, emergence

JEL Classification: 031, 033, D85 